\title{
FLÓRULA DA REGIÃO DO JARAU, MUNICÍPIO DE QUARAÍ - RIO GRANDE DO SUL ${ }^{1}$
}

\author{
FABIANO DA SILVA ALVES ${ }^{2}$ JOSÉ NEWTON CARDOSO MARCHIORI $^{3}$ \\ LEONARDO PAZ DEBLE ${ }^{4}$ ANABELA SILVEIRA DE OLIVEIRA-DEBLE 5
}

\section{RESUMO}

São listadas em ordem alfabética as famílias botânicas e espécies vegetais encontradas na Região do Jarau, município de Quaraí, oeste do Rio Grande do Sul. Informações adicionais sobre o hábito das plantas e tipologias de vegetação são também fornecidas.

Palavras-chave: Flora, Flórula, Jarau, Quaraí, Rio Grande do Sul.

\section{ABSTRACT}

[Florula of Jarau Region, municipality of Quarai - Rio Grande do Sul State, Brazil].

Botanical families and plant species growing in Jarau Region, west of Rio Grande do Sul State are listed in alphabetical order. Additional information related to plant habit and vegetation types are also given.

Key words: Flora, Florula, Jarau, Quarai, Rio Grande do Sul State, Brazil.

\section{INTRODUÇÃO}

Situada em pleno Planalto da Campanha, em terras do município de Quaraí, a "Região do Jarau" é definida neste trabalho por um polígono de $135,16 \mathrm{~km}^{2}$, limitado ao norte pelo arroio Garupá, ao sul pelo arroio Quaraí-Mirim e a leste e oeste por linhas imaginárias correspondentes às longitudes de $56^{\circ} 28^{\prime} 39^{\prime \prime} \mathrm{W}$ e $56^{\circ} 35^{\prime} 26^{\prime \prime} \mathrm{W}$. O principal acesso se dá pela rodovia estadual RS 377, distando, aproximadamente, $20 \mathrm{~km}$ ao norte da área urbana do município (Figura 1).

Visitada, inicialmente, por Friedrich Sellow, no primeiro quartel do século XIX, a Região do Jarau foi investigada no verão de 1944-1945 por Balduíno Rambo ${ }^{6}$ e, desde então, constitui ponto de coleta bastante conhecido. Da literatura es-

${ }_{1}$ Recebido em 10-11-2012 e aceito para publicação em 05-01-2013.

2 Dr. Professor do Curso de Ciências Biológicas, URCAMP (Alegrete - RS).

${ }^{3}$ Dr. Professor Titular do Departamento de Ciências Florestais, Universidade Federal de Santa Maria. Bolsista de Produtividade em Pesquisa ( $\mathrm{CNPq}$ - Brasil).

${ }^{4}$ Dr. Professor do Curso de Ciências da Natureza, UNIPAMPA (Dom Pedrito - RS).

5 Dra. Professora do Curso de Tecnólogo em Gestão Ambiental, URCAMP (Dom Pedrito - RS).

${ }^{6}$ RAMBO, A.B. Diário da segunda viagem de Balduino Rambo ao sudoeste do Rio Grande do Sul. Balduinia, Santa Maria, n. 38, p. 1-24, 2012. pecífica, salientam-se as contribuições de Alves (2012) e Alves \& Marchiori (2010a, b), sobre a fitogeografia e florística regionais.

\section{MATERIAL E MÉTODOS}

O trabalho foi realizado a partir de coletas periódicas nos anos de 2009 a 2011, cobrindo todas as estações. No campo, além da coleta de material fértil, realizou-se o registro fotográfico de cada uma das espécies, anotando-se, para as mesmas, informações variadas sobre o hábito e a(s) respectiva(s) tipologia(s) de vegetação.

\section{LISTA DAS ESPÉCIES}

As espécies da presente Flórula do Jarau são apresentadas a seguir, em ordem alfabética de famílias e gêneros. Na listagem, o hábito vegetal (HAB.), o status de ocorrência (OCOR.) e a respectiva tipologia de vegetação (TIPOL.), são indicados pelas seguintes convenções: er (erva), sub (subarbusto), arb (arbusto), arv (árvore), palm (palmeira), ep (epífita), trep (trepadeira), enr (enredadeira), h (hemiparasito), len (planta lenhosa), sbl (planta sublenhosa), an (planta anual), en (planta endêmica), nat (planta nativa), exot (planta exótica), cv (campo vulcânico), ca (campo em colinas de arenito), cr (campo rupestre), mc (mata ciliar), cm (capão-demato), pi (parque de inhanduvá). 


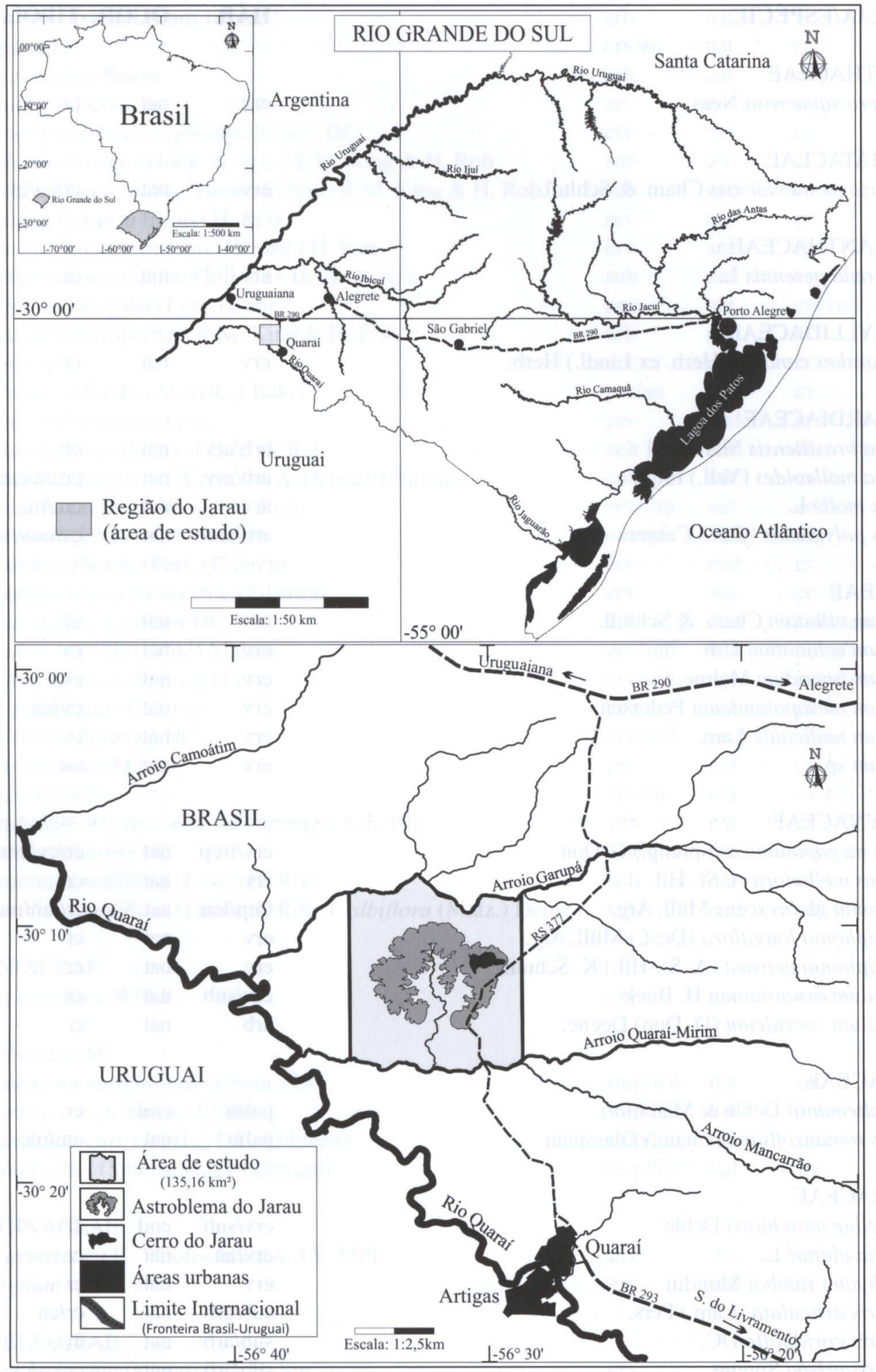

FIGURA 1 - Mapa de localização da Região do Jarau, Quaraí, Rio Grande do Sul (Brasil). 
FAMÍLIA/ESPÉCIE

HÁB. OCOR. TIPOL.

ACANTHACEAE

Dicliptera squarrosa Nees

erv nat cr

ALISMATACEAE

Sagittaria montevidensis Cham. \& Schltdl.

AMARANTHACEAE

Gomphrena perennis L.

AMARYLLIDACEAE

Zephyranthes candida (Herb. ex Lindl.) Herb.

ANACARDIACEAE

Lithraea brasiliensis Marchand

Lithraea molleoides (Vell.) Engl.

Schinus molle L.

Schinus polygamus (Cav.) Cabrera

\section{APIACEAE}

Eryngium ciliatum Cham. \& Schltdl.

Eryngium echinatum Urb.

Eryngium horridum Malme

Eryngium mesopotamicum Pedersen

Eryngium nudicaule Lam.

Eryngium sp.

\section{APOCYNACEAE}

Araujia megapotamica (Spreng.) G. Don

Asclepias mellodora A. St.-Hil.

Forsteronia glabrescens Müll. Arg.

Macrosiphonia longiflora (Desf.) Müll. Arg.

Macrosiphonia petraea (A. St.-Hil.) K. Schum.

Oxypetalum arnottianum $\mathrm{H}$. Buek

Oxypetalum coeruleum (D. Don) Decne.

\section{ARECACEAE}

Butia lallemantii Deble \& Marchiori

Syagrus romanzoffiana (Cham.) Glassman

\section{ASTERACEAE}

Achyrocline marchiorii Deble

Ambrosia elatior L.

Angelphytum ramboi Mondin

Baccharis articulata (Lam.) Pers.

Baccharis coridifolia DC.

Baccharis crispa Spreng.

Baccharis dracunculifolia DC. erv nat $\mathrm{cr} / \mathrm{ca} / \mathrm{cv}$

erv nat cr

erv nat cr

$\begin{array}{lll}\text { arb/arv } & \text { nat } & \mathrm{cr} \\ \text { arb/arv } & \text { nat } & \mathrm{cr} / \mathrm{cv} / \mathrm{cm} / \mathrm{mc} \\ \text { arv } & \text { nat } & \mathrm{cm} / \mathrm{mc} \\ \text { arb/arv } & \text { nat } & \mathrm{cv} / \mathrm{cm} / \mathrm{mc}\end{array}$

erv nat $\mathrm{cr}$

erv nat cv

erv nat cv

erv nat cv/ca

erv nat $\mathrm{cv}$

erv nat ca

erv/trep nat $\mathrm{cr} / \mathrm{cv} / \mathrm{cm} / \mathrm{mc}$

erv nat cr

trep/len nat $\mathrm{cr} / \mathrm{cm} / \mathrm{mc}$

erv nat $\mathrm{cr}$

erv nat cr

erv/sub nat cr

arb nat cr

palm end cr

palm nat $\mathrm{cm} / \mathrm{mc}$

$\begin{array}{lll}\text { erv/sub } & \text { end } & \mathrm{cr} \\ \text { erv/an } & \text { nat } & \mathrm{cr} \\ \text { erv } & \text { nat } & \mathrm{cr} \\ \text { sub/arb } & \text { nat } & \mathrm{cr} / \mathrm{ca} \\ \text { sub/arb } & \text { nat } & \mathrm{cv} \\ \text { sub/arb } & \text { nat } & \mathrm{cr} / \mathrm{ca} / \mathrm{cv} \\ \text { arb } & \text { nat } & \mathrm{cr}\end{array}$


Baccharis linearifolia (Lam.) Pers.

Bidens pilosa L.

Calea clematidea Baker

Calea uniflora Less.

Campuloclinium macrocephalum (Less.) DC.

Chromolaena hirsuta (Hook. \& Arn.) R.M. King \& H. Rob

Chromolaena squarrulosa (Hook. \& Arn.) R.M. King \& H. Rob.

Chrysolaena flexuosa (Sims) H. Rob.

Chrysolaena lithospermifolia (Hieron.) H. Rob.

Eupatorium tanacetifolium Gillies ex Hook. \& Arn.

Gochnatia polymorpha (Less.) Cabr.

Grazielia serrata (Spreng.) R.M. King \& H. Rob.

Hieracium sp.

Hysterionica nidorelloides (DC.) Baker

Isostigma crithmifolium Less.

Lessingianthus brevifolius (Less.) H. Rob.

Lessingianthus laniferus (Cristóbal \& Dematt.) Ângulo

Lessingianthus sellowii (Less.) H. Rob.

Mikania anethifolia (DC.) Matzenb.

Noticastrum diffusum (Pers.) Cabrera

Orthopappus angustifolius (Sw.) Gleason

Pterocaulon polystachyum DC.

Pterocaulon rugosum (Vahl) Malme

Senecio brasiliensis (Spreng.) Less.

Senecio pampeanus Cabrera

Senecio selloi (Spreng.) DC.

Solidago chilensis Meyen

Soliva sessilis Ruiz \& Pav.

Stenocephalum megapotamicum (Spreng.) Sch. Bip.

Verbesina sordescens DC.

Vernonanthura nudiflora (Less.) H. Rob.

Vernonanthura nudiflora (Less.) H. Rob. f. albiflora (Matz.) Deble

\section{BEGONIACEAE}

Begonia cucullata Willd.

erv nat $\mathrm{ca} / \mathrm{cv}$

\section{BIGNONIACEAE}

Adenocalymma marginatum (Cham.) DC.

Dolichandra cynanchoides Cham.

Dolichandra unguis-cati (L.) L.G. Lohmann

Tanaecium selloi (Spreng.) L.G. Lohmann

\section{BORAGINACEAE}

Cordia americana (L.) Gottschling \& J.S. Mill.

Heliotropium sp. trep/len

trep/len

trep/len

trep/len arb nat cr

erv/an nat cr

arb nat $\mathrm{cr}$

erv nat cr

erv nat cr

sub end cr

sub end cr

erv nat cr

erv nat cr

sub nat cr

arv nat $\mathrm{cm} / \mathrm{mc}$

arb nat cr

erv nat cr

erv/an nat cv

erv end cr

sub nat cr

sub nat cr

erv/sub nat $\mathrm{cr} / \mathrm{cv}$

ev/enr end cr

erv end cr

erv nat cr

erv end $\mathrm{cr} / \mathrm{ca} / \mathrm{cv}$

erv/sub nat $\mathrm{cr} / \mathrm{ca} / \mathrm{cv}$

erv/sub nat $\mathrm{cr} / \mathrm{ca} / \mathrm{cv}$

erv nat $\mathrm{cr} / \mathrm{ca}$

erv/sub end cr/ca

erv nat $\mathrm{cv}$

erv/an nat cv/ca

sub nat cr

erv/sub nat cr

sub nat cv

sub end $\mathrm{cr} / \mathrm{cv}$

arv nat

erv nat $\mathrm{cr}$

$\mathrm{cm} / \mathrm{mc}$

$\mathrm{cr}$

BROMELIACEAE

Aechmea recurvata (Klotzsch) L.B. Sm.

Dyckia vicentensis Strehl erv nat

$\mathrm{cr} / \mathrm{cm}$

erv

nat

cr 
Tillandsia aëranthos (Loisel.) L.B. Sm.

Tillandsia duratii Vis.

Tillandsia lorentziana Griseb.

Tillandsia recurvata (L.) L.

\section{BUDDLEJACEAE}

Buddleja thyrsoides Lam.

\section{CACTACEAE}

Cereus hildmannianus K. Schum.

Echinopsis rhodotricha K. Schum.

Frailea castanea Backeb.

Frailea fulviseta Buining \& Brederoo

Opuntia elata Salm-Dyck

Parodia ottonis (Lehm.) N. P. Taylor

Parodia oxicostata (Buining \& Brederoo) Hofacker

Rhipsalis aculeata F.A.C. Weber

Rhipsalis lumbricoides (Lem.) Lem. ex Salm-Dyck

CANNABACEAE

Celtis ehrenbergiana (Klotzsch) Liebm.

Celtis iguanaea (Jacq.) Sarg.

\section{CARDIOPTERIDACEAE}

Citronella gongonha (Mart.) R.A. Howard

\section{CARICACEAE}

Vasconcellea quercifolia A. St.-Hil.

\section{CELASTRACEAE}

Maytenus muelleri Schwacke

Schaefferia argentinensis Speg.

\section{COMMELINACEAE}

Commelina erecta $\mathrm{L}$.

\section{CONVOLVULACEAE}

Dichondra microcalyx (Hallier f.) Fabris

Evolvulus glomeratus Nees \& Mart.

Evolvulus sericeus $\mathrm{Sw}$.

Ipomoea cairica (L.) Sweet

\section{CUCURBITACEAE}

Cayaponia bonariensis (Mill.) Mart.Crov.

Cyclanthera hystrix (Gillies ex Hook. \& Arn.) Arn.

\section{CYPERACEAE}

Cyperus haspan L.

Fimbristylis autumnalis (L.) Roem. \& Schult.

$\begin{array}{lll}\text { erv/ep } & \text { nat } & \mathrm{cr} / \mathrm{cv} / \mathrm{mc} / \mathrm{pi} \\ \text { erv/ep } & \text { nat } & \mathrm{cr} / \mathrm{pi} \\ \text { erv/ep } & \text { nat } & \mathrm{cr} \\ \text { erv/ep } & \text { nat } & \mathrm{cr} / \mathrm{cv} / \mathrm{cm} / \mathrm{mc} / \mathrm{pi}\end{array}$

arb

nat cr

\section{arb/arv nat $\mathrm{cr} / \mathrm{cv} / \mathrm{cm} / \mathrm{pi}$}

erv end cr

erv end cv

erv end $\mathrm{cv}$

arb end $\mathrm{cr} / \mathrm{cv} / \mathrm{pi}$

erv nat $\mathrm{cr}$

erv end cr

erv/ep end pi

erv/ep nat $\mathrm{cm} / \mathrm{mc}$

arb/arv nat $\mathrm{cv} / \mathrm{cm} / \mathrm{mc}$

arb/arv nat $\mathrm{cm} / \mathrm{mc}$

arb/arv nat mc

arv nat $\mathrm{cm}$

arb/arv nat $\mathrm{cr} / \mathrm{cm} / \mathrm{mc}$

arb/arv nat $\mathrm{cm} / \mathrm{mc}$

erv nat cr

$\begin{array}{lll}\text { erv } & \text { nat } & \mathrm{cv} \\ \text { erv } & \text { nat } & \mathrm{cr} / \mathrm{ca} \\ \text { erv } & \text { nat } & \mathrm{cr} / \mathrm{ca} / \mathrm{cv} \\ \text { erv/en } & \text { nat } & \mathrm{cr} / \mathrm{cm} / \mathrm{mc}\end{array}$

erv/enr nat $\mathrm{cr} / \mathrm{cm}$

erv/enr nat $\mathrm{cr} / \mathrm{cm}$

erv nat cv

erv nat $\mathrm{cr} / \mathrm{ca}$ 
DROSERACEAE

Drosera brevifolia Pursh

$\begin{array}{lll}\text { erv/an } & \text { nat } & \text { ca } \\ & & \\ \text { erv/sub } & \text { nat } & \mathrm{cr} \\ \text { arb } & \text { end } & \mathrm{cr} \\ \text { erv/an } & \text { nat } & \mathrm{cr} \\ \text { arb/arv } & \text { nat } & \mathrm{mc} \\ \text { arb/arv } & \text { nat } & \mathrm{cm} / \mathrm{mc} \\ \text { arb/arv } & \text { nat } & \mathrm{cm} / \mathrm{mc} \\ \text { arb/arv } & \text { nat } & \mathrm{cr} / \mathrm{cv} / \mathrm{cm} / \mathrm{mc} \\ \text { arb } & \text { nat } & \mathrm{mc} \\ \text { sub } & \text { nat } & \mathrm{cr}\end{array}$

\section{EUPHORBIACEAE}

Acalypha communis Müll. Arg.

Croton allemii G.L. Webster

Croton argenteus $\mathrm{L}$.

Manihot grahamii Hook.

Sapium haematospermum Müll. Arg.

Sebastiania brasiliensis Spreng.

Sebastiania commersoniana (Baill.) L.B. Sm. \& Downs

Sebastiania schottiana (Müll. Arg.) Müll. Arg.

Sebastiania serrulata (Mart.) Müll. Arg.

sub

$\begin{array}{lll}\text { erv } & \text { nat } & \mathrm{cr} \\ \text { erv } & \text { end } & \mathrm{cr} \\ \text { arb } & \text { nat } & \mathrm{cm} / \mathrm{mc} \\ \text { trep/len } & \text { end } & \mathrm{mc} \\ \text { erv/enr } & \text { nat } & \mathrm{cr} \\ \text { erv/sub } & \text { nat } & \mathrm{cr} \\ \text { erv/sub } & \text { nat } & \mathrm{cr} / \mathrm{ca} \\ \text { erv/sub } & \text { end } & \mathrm{cr} \\ \text { erv/sub } & \text { nat } & \mathrm{cr} \\ \text { erv/sub } & \text { end } & \mathrm{cr} \\ \text { erv/sub } & \text { nat } & \mathrm{cr} \\ \text { erv } & \text { nat } & \mathrm{cr} / \mathrm{ca} / \mathrm{cv} \\ \text { erv } & \text { nat } & \mathrm{cr} \\ \text { erv } & \text { nat } & \mathrm{cr} \\ \text { arb/arv } & \text { nat } & \mathrm{mc} \\ \text { erv/sub } & \text { nat } & \mathrm{cr} \\ \text { arv } & \text { nat } & \mathrm{cv} / \mathrm{cm} / \mathrm{mc} \\ \text { erv } & \text { nat } & \mathrm{cr} \\ \text { erv } & \text { end } & \mathrm{cr} \\ \text { erv } & \text { end } & \mathrm{cr} \\ \text { erv } & \text { end } & \mathrm{cr} \\ \text { erv } & \text { end } & \mathrm{cr} \\ \text { erv/an } & \text { end } & \mathrm{cr} \\ \text { erv } & \text { end } & \mathrm{cr} / \mathrm{ca} \\ \text { erv } & \text { nat } & \mathrm{cr} / \mathrm{ca} \\ \text { sub/arb } & \text { nat } & \mathrm{cv} \\ \text { arb } & \text { nat } & \mathrm{cv} \\ \text { arb } & \text { nat } & \mathrm{cr} \\ \text { erv/sub } & \text { nat } & \mathrm{cr} \\ \text { erv/sub } & \text { nat } & \mathrm{cr} \\ \text { sub } & \text { end } & \mathrm{cv} \\ \text { arv } & \text { nat } & \mathrm{cm} / \mathrm{mc} \\ \text { arb/arv } & \text { nat } & \mathrm{cv} / \mathrm{cm} / \mathrm{mc} \\ \text { sub } & \text { nat } & \mathrm{cr} \\ & & \end{array}$

FABACEAE

Aeschynomene histrix Poir.

Arachis burkartii Handro

Calliandra tweedii Benth.

Camptosema rubicundum Hook. \& Arn.

Centrosema virginianum (L.) Benth.

Chamaecrista repens (Vogel) H.S. Irwin \& Barneby

Chamaecrista rotundifolia (Pers.) Greene

Clitoria nana Benth.

Desmanthus virgatus (L.) Willd.

Desmodium arechavaletae Burkart

Desmodium cuneatum Hook. \& Arn.

Desmodium incanum DC.

Desmodium sp.

Eriosema tacuaremboense Arechav.

Erythrina cristagalli $\mathrm{L}$.

Galactia neesii DC.

Gleditsia amorphoides (Griseb.) Taub.

Indigofera asperifolia Bong. ex Benth.

Lathyrus nervosus Lam.

Lathyrus pubescens Hook. \& Arn.

Lathyrus subulatus Lam.

Lupinus albescens Hook. \& Arn.

Lupinus gibertianus C.P. Sm.

Macroptilium arenarium (Bacig.) S.I. Drewes \& R.A. Palacios

Macroptilium prostratum (Benth.) Urb.

Mimosa adpressa Hook. \& Arn.

Mimosa amphigena Burkart

Mimosa daleoides Benth.

Mimosa dolens Vell.

Mimosa flagellaris Benth.

Mimosa ramboi Burkart

Parapiptadenia rigida (Benth.) Brenan

Parkinsonia aculeata $\mathrm{L}$.

Poiretia tetraphylla (Poir.) Burkart 
Prosopis affinis Spreng.

Rhynchosia corylifolia Mart. ex Benth.

Rhynchosia diversifolia Micheli

Senegalia bonariensis (Gill. ex Hook. \& Arn.) Seigler \& Ebinger

Stylosanthes leiocarpa Vogel

Trifolium polymorphum Poir.

Vachellia caven (Molina) Seigler \& Ebinger

Vigna peduncularis (Kunth) Fawc. \& Rendle

Zornia latifolia Sm.

Zornia sp.

$\begin{array}{lll}\text { arv } & \text { nat } & \mathrm{pi} \\ \text { erv } & \text { nat } & \mathrm{cr} \\ \text { erv } & \text { nat } & \mathrm{cv} \\ \text { arb/arv } & \text { nat } & \mathrm{cr} / \mathrm{cv} / \mathrm{cm} / \mathrm{mc} \\ \text { erv/sub } & \text { nat } & \mathrm{cr} \\ \text { erv } & \text { nat } & \mathrm{cr} / \mathrm{ca} / \mathrm{cv} \\ \text { arb/arv } & \text { nat } & \mathrm{cv} / \mathrm{cm} / \mathrm{mc} \\ \text { erv/enr } & \text { nat } & \mathrm{cr} \\ \text { erv } & \text { nat } & \mathrm{cr} \\ \text { erv } & \text { nat } & \mathrm{cr} / \mathrm{ca}\end{array}$

HYPERICACEAE

Hypericum connatum Lam.

erv/sub nat cr

IRIDACEAE

Calydorea alba Roitman \& A. Castillo

$\begin{array}{lll}\text { erv } & \text { nat } & \mathrm{cr} \\ \text { erv } & \text { end } & \mathrm{cr} \\ \text { erv } & \text { end } & \mathrm{cr} / \mathrm{ca} \\ \text { erv } & \text { end } & \mathrm{cv} \\ \text { erv } & \text { end } & \mathrm{cr} \\ \text { erv } & \text { end } & \mathrm{cr} \\ \text { erv } & \text { end } & \mathrm{cv} \\ \text { erv } & \text { end } & \text { ca }\end{array}$

Calydorea approximata R.C. Foster

Cypella fucata Ravenna

Cypella herbertii (Lindl.) Herb.

Cypella sp.1

Cypella sp.2

Herbertia lahue (Molina) Goldblatt

Onira unguiculata (Backer) Ravena

erv

end ca

\section{LAMIACEAE}

Glechon thymoides Spreng.

erv/sub end cr

LAURACEAE

Nectandra megapotamica (Spreng.) Mez

arv

nat $\mathrm{cm} / \mathrm{mc}$

LOGANIACEAE

Strychnos brasiliensis (Spreng.) Mart.

arv/arb nat mc

\section{LORANTHACEAE}

Ligaria cuneifolia (Ruiz \& Pav.) Tiegh.

Tripodanthus acutifolius (Ruiz \& Pav.) Tiegh.

$\mathrm{h}$

$\mathrm{h}$

nat

nat

$\mathrm{cv} / \mathrm{cm} / \mathrm{mc}$

$\mathrm{cv} / \mathrm{cm} / \mathrm{mc}$

\section{LYTHRACEAE}

Heimia myrtifolia Cham. \& Schltdl.

sub/arb nat

$\mathrm{cv} / \mathrm{cm} / \mathrm{mc}$

Heimia salicifolia (Kunth) Link

sub/arb nat

$\mathrm{cv} / \mathrm{cm} / \mathrm{mc}$

\section{MALPIGHIACEAE}

Callaeum psilophyllum (A. Juss.) D.M. Johnson

Janusia guaranitica (A. St.-Hil.) A. Juss.

trep/len nat

trep/sbl nat

$\begin{array}{lll}\text { sub/arb } & \text { nat } & \mathrm{cr} \\ \text { arv } & \text { nat } & \mathrm{cm} / \mathrm{mc} \\ \text { erv/sub } & \text { nat } & \mathrm{cr} \\ \text { erv/sub } & \text { nat } & \mathrm{cr}\end{array}$

erv/sub nat cr

\section{MALVACEAE}

Abutilon grandifolium (Willd.) Sweet

Luehea divaricata Mart. \& Zucc.

Melochia chamaedrys A. St.-Hil.

Pavonia hastata Cav. 
Pavonia sepium A. St.-Hil.

Sida $\mathrm{sp.}$

MELASTOMATACEAE

Miconia hyemalis A. St.-Hil. \& Naudin

Tibouchina gracilis (Bonpl.) Cogn.

MELIACEAE

Trichilia elegans A. Juss.

MORACEAE

Ficus luschnathiana (Miq.) Miq.

MYRTACEAE

Blepharocalyx salicifolius (Kunth) O. Berg

Eugenia uniflora L.

Myrceugenia myrtoides O. Berg

Myrcia selloi (Spreng.) N. Silveira

Myrcianthes cisplatensis (Cambess.) O. Berg

Myrcianthes pungens (O.Berg) D. Legrand

Psidium incanum (O. Berg) Burret

Psidium luridum (Spreng.) Burret

ONAGRACEAE

Oenothera affinis Cambess.

ORCHIDACEAE

Oncidium bifolium (Sims) Dumort

Amphiglossum sp.

OXALIDACEAE

Oxalis conorrhiza Jacq.

Oxalis sp.1

Oxalis sp. 2

PASSIFLORACEAE

Passiflora caerulea L.

PHYLLANTHACEAE

Phyllanthus sellowianus (Klotzsch) Müll. Arg.

PLANTAGINACEAE

Angelonia integerrima Spreng.

POACEAE

Andropogon lateralis Nees

Andropogon macrothrix Trin.

Andropogon selloanus (Hack.) Hack.

Aristida circinalis Lindm. arb nat cr

erv/sub nat cr

arb/arv nat cr

erv/sub nat $\mathrm{cr} / \mathrm{ca}$

arb/arv nat me

arv nat $\mathrm{cr} / \mathrm{cm} / \mathrm{mc}$

$\begin{array}{lll}\text { arb/arv } & \text { nat } & \mathrm{cv} / \mathrm{cm} / \mathrm{mc} \\ \text { arb/arv } & \text { nat } & \mathrm{cm} / \mathrm{mc} \\ \text { arb } & \text { nat } & \mathrm{cr} \\ \text { arb/arv } & \text { nat } & \mathrm{mc} \\ \text { arb/arv } & \text { nat } & \mathrm{cv} / \mathrm{cm} / \mathrm{mc} \\ \text { arv } & \text { nat } & \mathrm{cm} / \mathrm{mc} \\ \text { sub } & \text { nat } & \mathrm{cr} \\ \text { sub } & \text { nat } & \mathrm{cr}\end{array}$

erv/an nat cv

erv/ep nat $\mathrm{cm} / \mathrm{mc}$

erv nat $\mathrm{cr} / \mathrm{cv}$

erv nat cr

erv nat $\mathrm{cr} / \mathrm{ca} / \mathrm{cv}$

erv nat $\mathrm{cr} / \mathrm{ca} / \mathrm{cv}$

trep nat $\mathrm{cr} / \mathrm{cv} / \mathrm{cm} / \mathrm{mc}$

arb nat mc

erv nat cr

$\begin{array}{lll}\text { erv } & \text { nat } & \mathrm{ca} / \mathrm{cv} \\ \text { erv } & \text { nat } & \mathrm{cr} \\ \text { erv } & \text { nat } & \mathrm{cr} / \mathrm{ca} / \mathrm{cv} \\ \text { erv } & \text { nat } & \mathrm{cr} / \mathrm{ca}\end{array}$


Aristida filifolia (Arechav.) Herter

Aristida venustula Arechav.

Axonopus affinis Chase

Axonopus argentinus Parodi

Bothriochloa laguroides (DC.) Herter

Chusquea ramosissima Lindm.

Digitaria aequiglumis (Hack. \& Arechav.) Parodi

Eleusine tristachya (Lam.) Lam.

Elionurus muticus (Spreng.) Kuntze

Eragrostis airoides Nees.

Eragrostis bahiensis Schrad. ex Schult.

Eragrostis lugens Nees

Eragrostis neesii Trin.

Eragrostis plana Nees

Eustachys polystachya (Lag.) Kunth

Leptocoryphium lanatum (Kunth) Nees

Melinis repens (Willd.) Zizka

Panicum sp.

Paspalum ammodes Trin.

Paspalum dilatatum Poir.

Paspalum notatum Flüggé

Paspalum plicatulum Michx.

Paspalum polyphyllum Nees ex Trin.

Paspalum stellatum Humb. \& Bonpl. ex Flüggé

Paspalum urvillei Steud.

Rhynchelitrum repens (Wild.) C.E.Hubb.

Schizachyrium condensatum (Kunth) Nees

Schizachyrium microstachyum (Desv. ex Ham.) Roseng.

Schizachyrium sp.

Setaria parviflora (Poir.) Kerguélen

Spartina sp.

Sporobolus indicus (L.) R. Br.

Tripogon spicatus (Nees) Ekman

\section{POLYGONACEAE}

Coccoloba argentinensis Speg.

Ruprechtia laxiflora Meisn.

Ruprechtia salicifolia (Cham. \& Schltdl.) C.A.Mey.

\section{POLYPODIACEAE}

Microgramma sp.

\section{PRIMULACEAE}

Myrsine laetevirens (Mez) Arechav.

Myrsine lorentziana (Mez.) Arechav.

\section{RHAMNACEAE}

Discaria americana Gillies \& Hook.

Scutia buxifolia Reissek

$\begin{array}{lll}\text { erv } & \text { nat } & \mathrm{cr} / \mathrm{ca} \\ \text { erv } & \text { nat } & \mathrm{cr} / \mathrm{ca} \\ \text { erv } & \text { nat } & \mathrm{cr} / \mathrm{ca} \\ \text { erv } & \text { nat } & \mathrm{cr} / \mathrm{ca} \\ \text { erv } & \text { nat } & \mathrm{cr} / \mathrm{ca} / \mathrm{cv} \\ \text { erv/sbl } & \text { nat } & \mathrm{mc} \\ \text { erv/an } & \text { nat } & \mathrm{cr} / \mathrm{ca} \\ \text { erv/an } & \text { nat } & \mathrm{cr} / \mathrm{ca} / \mathrm{cv} \\ \text { erv } & \text { nat } & \mathrm{cr} \\ \text { erv } & \text { nat } & \mathrm{cr} \\ \text { erv } & \text { nat } & \mathrm{cr} / \mathrm{ca} \\ \text { erv } & \text { nat } & \mathrm{cr} \\ \text { erv } & \text { nat } & \mathrm{cr} / \mathrm{ca} / \mathrm{cv} \\ \text { erv } & \text { exot } & \mathrm{cr} / \mathrm{ca} / \mathrm{cv} \\ \text { erv } & \text { nat } & \mathrm{cr} \\ \text { erv } & \text { nat } & \mathrm{cr} \\ \text { erv } & \text { exot } & \mathrm{cr} \\ \text { erv } & \text { nat } & \mathrm{cr} \\ \text { erv } & \text { nat } & \mathrm{cr} / \mathrm{ca} \\ \text { erv } & \text { nat } & \mathrm{cr} / \mathrm{ca} \\ \text { erv } & \text { nat } & \mathrm{cr} / \mathrm{ca} / \mathrm{cv} \\ \text { erv } & \text { nat } & \mathrm{cr} / \mathrm{ca} \\ \text { erv } & \text { nat } & \mathrm{cr} / \mathrm{ca} \\ \text { erv } & \text { nat } & \mathrm{cr} \\ \text { erv } & \text { nat } & \mathrm{cr} / \mathrm{ca} \\ \text { erv } & \text { exot } & \mathrm{cr} / \mathrm{ca} \\ \text { erv } & \text { nat } & \mathrm{cr} / \mathrm{ca} \\ \text { erv } & \text { nat } & \mathrm{cr} / \mathrm{ca} \\ \text { erv } & \text { nat } & \mathrm{cr} / \mathrm{ca} \\ \text { erv } & \text { nat } & \mathrm{cr} / \mathrm{ca} \\ \text { erv } & \text { nat } & \mathrm{ca} / \mathrm{pi} \\ \text { erv } & \text { nat } & \mathrm{cr} / \mathrm{ca} \\ \text { erv } & \text { nat } & \mathrm{ca} / \mathrm{pi} \\ & & \\ \end{array}$

arb/arv nat mc

arv nat $\mathrm{cm} / \mathrm{mc}$

arb/arv nat mc

erv/ep nat $\mathrm{cm} / \mathrm{mc}$

$\begin{array}{lll}\text { arv } & \text { nat } & \mathrm{cr} / \mathrm{cm} / \mathrm{mc} \\ \text { arv } & \text { nat } & \mathrm{mc} \\ \text { arb } & \text { nat } & \mathrm{cv} \\ \text { arb/arv } & \text { nat } & \mathrm{cv} / \mathrm{cm} / \mathrm{mc}\end{array}$


arb/arv nat $\mathrm{cm} / \mathrm{mc}$

\section{RUBIACEAE}

Cephalanthus glabratus (Spreng.) K. Schum.

Galianthe fastigiata Griseb.

Galianthe longifolia (Standl.) E.L. Cabral

Guettarda uruguensis Cham. \& Schltdl.

Mitracarpus sp.

Richardia brasiliensis Gomes

$\begin{array}{lll}\text { arb } & \text { nat } & \mathrm{cr} / \mathrm{mc} \\ \text { erv/sub } & \text { nat } & \mathrm{cr} \\ \text { sub } & \text { nat } & \mathrm{cr} \\ \text { arb } & \text { nat } & \mathrm{cm} / \mathrm{mc} \\ \text { erv } & \text { nat } & \mathrm{cr} \\ \text { erv } & \text { nat } & \mathrm{cr}\end{array}$

\section{RUTACEAE}

Zanthoxylum fagara (L.) Sarg.

arv nat

$\mathrm{cv} / \mathrm{cm} / \mathrm{mc}$

Zanthoxylum rhoifolium Lam.

arv

nat

$\mathrm{cv} / \mathrm{cm} / \mathrm{mc}$

\section{SALICACEAE}

Casearia sylvestris $\mathrm{Sw}$.

arb/arv nat

$\mathrm{cm} / \mathrm{mc}$

Salix humboldtiana Willd.

Xylosma tweediana (Clos) Eichler

ar

arb/arv nat

$\mathrm{mc}$

SANTALACEAE

Acanthosyris spinescens (Mart. \& Eichler) Griseb.

Jodina rhombifolia (Hook. \& Arn.) Reissek

arv

$\operatorname{arv}$

nat

$\mathrm{mc}$

SAPINDACEAE

Allophylus edulis (A. St.-Hil., A. Juss. \& Camb.) Hier. ex Nied.

arb/arv nat

Allophylus guaraniticus (A. St.-Hil.) Radlk.

Cupania vernalis Cambess.

arb/arv nat

arv nat

Matayba elaeagnoides Radlk.

arb/arv nat

Urvillea uniloba Radlk.

trep/len nat

\section{SAPOTACEAE}

Chrysophyllum marginatum (Hook. \& Arn.) Radlk.

Pouteria salicifolia (Spreng.) Radlk.

arb/arv

nat

arb/arv nat

$\mathrm{cr} / \mathrm{cm} / \mathrm{mc}$

$\mathrm{mc}$

\section{SMILACACEAE}

Smilax campestris Griseb.

trep/len nat $\mathrm{cr} / \mathrm{cv} / \mathrm{cm} / \mathrm{mc}$

\section{SOLANACEAE}

Solanum laxum Spreng.

enr/trep end mc

\section{THYMELAEACEAE}

Daphnopsis racemosa Griseb.

arb

nat $\mathrm{cm} / \mathrm{mc}$

\section{VERBENACEAE}

Aloysia gratissima (Gillies \& Hook. ex Hook.) Tronc.

Citharexylum montevidense (Spreng.) Moldenke

Lippia arechavaletae Moldenke

Lippia coarctata Tronc.

Verbena intermedia Gillies \& Hook. ex Hook.

Verbena rigida Spreng.

$\begin{array}{lll}\text { arb } & \text { nat } & \mathrm{cv} / \mathrm{cm} / \mathrm{mc} \\ \text { arv } & \text { nat } & \mathrm{cm} / \mathrm{mc} \\ \text { sub } & \text { end } & \mathrm{cv} \\ \text { erv } & \text { end } & \mathrm{cr} \\ \text { erv } & \text { end } & \mathrm{cr} \\ \text { erv } & \text { nat } & \mathrm{cr}\end{array}$




\section{CONSIDERAÇÕES FINAIS}

Na Região do Jarau foram reconhecidas seis tipologias de vegetação natural, muito distintas entre si pela composição florística, fisionomia e aspectos fitoecológicos (campo rupestre, campo vulcânico, campo em colinas de arenito, mata ciliar, capões-de-mato e parque de inhanduvá), e identificadas 275 espécies botânicas, pertencentes a 62 famílias.

Trata-se de área de notável diversidade florística para o Planalto da Campanha, fato que se pode atribuir aos variados habitats decorrentes de uma movimentada geomorfologia (Cerros do Jarau), bem como às diferentes litologias e solos, inclusive no tocante à disponibilidade hídrica. A respeito desta flórula, salienta-se a sua riqueza em espécies endêmicas, raras e/ou ameaçadas de extinção, aspecto que recomenda a área em estudo como prioritária em uma rede de unidades de conservação do Bioma Pampa. Das 40 espécies consideradas endêmicas, 29 são do campo rupestre, fato que se explica pela diversidade de nichos ecológicos ali existentes. Mesmo assim, também se encontram endemismos em outras tipologias e, inclusive, no campo de colinas vulcânicas ${ }^{7}$, que tem "a flora mais pobre que se pode imaginar", de acordo com registro de Balduíno Rambo para a região ${ }^{8}$.

Mais de um terço das espécies (120) pertence às três famílias mais numerosas na flora campestre no Rio Grande do Sul: Fabaceae (44 espécies), Asteraceae (39) e Poaceae (37). Apesar dessa concentração, há que se ressaltar o grande número de famílias botânicas representadas na área em estudo.
Mesmo em exame superficial, salta aos olhos a predominância de espécies herbáceas e subarbustivas. O contingente lenhoso, apesar disso, se mostra expressivo em todas as tipologias, e não apenas nas matas ciliares e capões-de-mato, mas, inclusive, nas formações campestres. A lista, por fim, inclui três espécies exóticas de Poaceae (Eragrostis plana, Melinis repens e Rhynchelitrum repens), a primeira das quais, de grande potencial invasor, é bem conhecida por seu difícil controle.

\section{REFERÊNCIAS BIBLIOGRÁFICAS}

ALVES, F. da S. Fitogeografia da Região do Jarau - Quaraí/RS. 2012. 110 p. Tese (Doutorado em Engenharia Florestal) - Universidade Federal de Santa Maria, Santa Maria, 2012.

ALVES, F. da S.; MARCHIORI, J.N.C. Nota sobre a ocorrência natural do butiá-anão (Butia lallemantii Deble \& Marchiori) no Cerro do Jarau, Quaraí - Rio Grande do Sul. Balduinia, Santa Maria, n. 24, p. 29-31, 2010a.

ALVES, F. da S.; MARCHIORI, J.N.C. O inhanduvá (Prosopis affinis Spreng.) no Rio Grande do Sul. 2 - Ocorrência natural na região do Jarau, Quaraí. Balduinia, Santa Maria, n. 25, p. 1-9, $2010 \mathrm{~b}$.

BURKART, A. Leguminosas nuevas o criticas. Darwiniana, San Isidro, v. 7, n. 2, p. 216-239, 1946.

RAMBO, A.B. Diário da segunda viagem de Balduino Rambo ao sudoeste do Rio Grande do Sul. Balduinia, Santa Maria, n. 38, p. 1-24, 2012. RAMBO, B. A Fisionomia do Rio Grande do Sul. Ensaio de Monografia Natural. Porto Alegre: Of. Graf. da Imprensa Oficial, 1942. 360 p.

7 É o caso de Mimosa ramboi, por exemplo, cujo tipo procede, justamente, da região do Jarau (ver BURKART, 1946, p. 229).

8 RAMBO, B. A Fisionomia do Rio Grande do Sul. Ensaio de Monografia Natural. Porto Alegre: Of. Graf. da Imprensa Oficial, 1942. p. 115. 